\title{
Utilization and uptake of the UpToDate clinical decision support tool at the Makerere University College of Health Sciences (MakCHS), Uganda
}

\author{
Alison Annet Kinengyere ${ }^{1}$, Julie Rosenberg ${ }^{2}$, Olivia Pickard ${ }^{2}$, Moses Kamya ${ }^{3}$
}

1. Makerere University College of Health Sciences Library.

2. Ariadne Labs, Brigham and Women's Hospital

3. Makerere University College of Health Sciences.

\begin{abstract} in Africa. infectious diseases. Access occurred mainly through the mobile phone app.

DOI: https://dx.doi.org/10.4314/ahs.v21i2.52 abs.v21i2.52

\section{Background}

Digital point-of-care tools can provide easy-to-use, high-quality information that is regularly updated in line with the science and have been shown to improve diagnostic accuracy and promote quality, efficient care ${ }^{1}$. The use of such tools is becoming increasingly popular throughout the world. A majority of the increased use is happening in North America, though Asia Pacific is anticipated to be the fastest growing region in adopting these tools ${ }^{2}$. An American Medical Association survey showed that $57 \%$ of physicians use or plan to use a digital clinical support tool in their work ${ }^{3}$. The clinical decision support resource UpToDate is used in over 190 countries and by $90 \%$ of US academic medical centers ${ }^{4}$.
\end{abstract}

Background: The use of point-of-care, evidence-based tools is becoming increasingly popular. They can provide easy-touse, high-quality information which is regularly updated and has been shown to improve clinical outcomes. Integrating such tools into clinical practice is an important component of improving the quality of health care. However, because such tools are rarely used in resource-limited settings, there is limited research on uptake especially among medical students.

Objective: This paper explores the uptake of one such tool, Up-To-Date, when provided free of cost at a medical school

Methods: In partnership with the Better Evidence at Ariadne Labs free access to UpToDate was granted through the MakCHS IP address. On-site librarians facilitated training sessions and spread awareness of the tool. Usage data was aggregated, based on log ins and content views, presented and analyzed using Excel tables and graphs.

Results: The data shows evidence of meaningful usage, with 43,043 log ins and 15,591 registrations between August 2019 and August 2020. The most common topics viewed were in obstetrics and gynecology, pediatrics, drug information, and

Conclusion: Findings show usage by various user categories, but with inconsistent uptake and low usage. Librarians can draw upon these results to encourage institutions to support uptake of point-of-care tools in clinical practice.

Keywords: UpToDate clinical decision support tool, Makerere University College of Health Sciences, Uganda.

Cite as: Kinengyere $A A$, Rosenberg J, Pickard O, Kamya M. Utilization and uptake of the UpToDate clinical decision support tool at the Makerere University College of Health Sciences (MakCHS), Uganda. Afri Health Sci. 2021;21(2). 904-911. https: / dx.doi.org/ 10.4314/

\section{Corresponding author: \\ Alison Annet Kinengyere, \\ Makerere University College of Health \\ Sciences, Library \\ Email: alison.kinengyere@mak.ac.ug}

Integrating these tools into practice, particularly in sub-Saharan Africa, is an important component of improving the overall quality of healthcare. However, significant gaps exist in the implementation of these tools into healthcare providers' routine practice in low-resource settings ${ }^{5}$. Though medical students in the US are likely to be introduced to these digital tools early in their career ${ }^{6}$, the use of digital tools has not gained the same momentum in sub-Saharan African medical education ${ }^{7}$. Research conducted by the authors of this study at the University of Rwanda suggests that early introduction of an evidence-based tool to medical students leads to habit formation and use of the tool in later clinical practice ${ }^{8}$. However, there has been limited research on this topic, likely due to limited access to such tools whose cost can be prohibitive. Sub-Saharan African medical schools have faced insufficient access to digital clinical resources, with medical schools rating their technological resources somewhat to severely inadequate on average? 


\section{About UpToDate}

UpToDate is a clinical decision support resource that can be used on or offline and provides evidence-based information for medical doctors. It can be accessed on digital devices such as computers, tablets, or mobile smart phones in hospitals, clinics, or homes. The tool is authored by 7,100 physicians who continuously synthesize the most recent medical information across specialties into evidence-based recommendations that can support point-of-care decisions. UpToDate is used in over 190 countries and by $90 \%$ of US academic medical centers ${ }^{4}$.

Recent research demonstrates that the use of UpToDate was associated with improved quality of care, shorter lengths of stay, and lower mortality rates over a three-year period ${ }^{1}$. UpToDate has been shown to answer clinical questions effectively, with one study citing an $86 \%$ answer retrieval rate for UpToDate ${ }^{10}$. In medical education, UpToDate is reported to be a highly effective resource for learning ${ }^{11}$ and is preferred by early career doctors ${ }^{12}$.

\section{UpToDate Content}

The tool covers a range of information areas and tools, including: topic updates by specialty, clinical calculators, drug interaction checkers, and search functionality (by disease name, symptom, lab abnormality, procedure, or drug) with filter options (adult, pediatric, or patient graphics). While users can search UpToDate in many languages, the content is available only in English. With the emergence of the COVID-19 pandemic, UpToDate has added new open-access information covering clinical topics, questions, patient education and society guidelines. The pace of discovery and science related to the COVID-19 pandemic as well as the spread of misinformation, coupled with shortages of health workers have only hastened the need for clinicians to have evidence-based and trusted resources to which they can turn for clinical information.

\section{Accessing UpToDate}

UpToDate is a commercial product available for purchase. Better Evidence, a group at Ariadne Labs - a joint center for health systems innovations at Harvard T.H. Chan School of Public Health and Brigham and Women's Hospital - works to facilitate access to evidence-based clinical resources to health providers serving vulnerable populations who couldn't otherwise afford them. A pilot study run by Better Evidence demonstrated the utility of Up-To-Date among medical students at University of Rwanda ${ }^{8}$, the group be- gan facilitating donated institutional licenses to medical schools across Africa as part of the Better Evidence for training program. Access was granted to MakCHS in 2019, with plans to add new schools annually.

With an institutional license, users can access UpToDate (www.uptodate.com) on the institutional local area network (LAN) and register for an individual account that will allow them to access UpToDate outside the LAN and download the content for use offline. The ability to use the tool offline is a valuable feature in developing countries.

\section{About the study site (MakCHS) \\ Established in 1922 as a technical school, Makerere Uni- versity is one of the oldest and most prestigious English Universities in Africa. The college soon began offering various other courses in medical care, agriculture, veter- inary sciences and teacher training. It expanded over the years to become a center for higher education in East Africa in $1935^{13}$.}

On July 1, 1970, Makerere became an independent national university of the Republic of Uganda, offering undergraduate and postgraduate courses. Makerere University offers not only day but also evening and external study programmes to a student body of about 35,000 undergraduates and 3,000 postgraduates (both Ugandan and foreign).

The university transitioned from the faculty-based to the collegiate system in 2011. As of July 2014, it includes 10 constituent colleges including the School of Law, all operating as semi-autonomous units ${ }^{13}$.

Makerere University College of Health Sciences was transformed from a Faculty of Medicine into a College in 2013. It is comprised of 4 schools (Medicine, Biomedical Sciences, Public Health and Health Sciences) and 27 departments, with a total population of 3,018 students, 249 academic staff, who double as lecturers and health workers, mainly stationed at the Mulago National Referral and Teaching Hospital. It is this population, together with other health researchers who are affiliated to the College that utilizes the UpToDate subscription facilitated by Better Evidence to support clinical practice ${ }^{14}$.

\section{Objective}

This paper explores the uptake of UpToDate among medical students and faculty at Makerere University College of Health Sciences. 


\section{Methodology}

After being approved for participation in the Better Evidence for training program, MakCHS entered into a contract with UpToDate. UpToDate established access to the product through the LAN at the university and its affiliated training facilities in August 2019. Better Evidence team members aided librarians, students, and faculty at MakCHS in learning how to access, register for, and use the tool. The MakCHS librarians then communicated about the tool and built awareness of the tool in their respective institutions through training sessions on campus and messaging.

UpToDate tracked aggregate usage and searches by registered, logged-in users or users on campus and shared this data with the school through the Better Evidence program every two months. Usage of the open-access information on COVID-19 was not tracked if users were offsite and not logged in. No institutional review board approvals were needed given the nature of this work and the aggregate nature of the data that did not allow any individuals to be identified.

The data presented here captured usage information from August 2019 to August 2020 on the following:

a) Trending topics sorted by most views-topics that increased in popularity from the prior two month period

b) Methods used to access the topics-whether users accessed UpToDate via the website on a computer or via the UpToDate app on their smart phones, tablets or iPads

c) Roles of the users that accessed UpToDate

d) Top five medical topics by views - the most frequently visited topic cards during each two-month period

e) Top five medical specialties viewed - what specialties the topics viewed fall into

f) Total usage by month — how many searches were done

\section{Data analysis}

We used Excel to organize the data into tables and graphs to enable us to look at the trends and usage patterns over time. We then grouped the relevant data based on trending topics, access methods, user roles, topic specialties by views, medical topics by views and total usage of the tool by month. Reporting periods are labeled by when the report was received. UpToDate provided reports every two months.

\section{Results}

\section{Trending Topics}

Table 1 shows the trending topics sorted by increase in views for each two-month reporting period. More recently trending topics have been related to one another. In general, trends show the wide array of topics viewed and how they can change from month to month.

Table 1: Trending Topics

\begin{tabular}{|c|l|}
\hline Month of report & Topic viewed \\
\hline Aug-20 & Approach to the adult with acute diarrhea in resource-rich settings \\
\hline & Preeclampsia: Clinical features and diagnosis \\
\hline & Spontaneous abortion: management \\
\hline Jun-20 & Convulsive status epilepticus in adults: Treatment and prognosis \\
\hline & $\begin{array}{l}\text { Convulsive status epilepticus in adults: Classification, clinical } \\
\text { features, and diagnosis }\end{array}$ \\
\hline & Acute liver in children: Management, complications, and outcomes \\
\hline Apr-20 & Valvar lesions: differential diagnosis based on morphology \\
\hline & Biliary atresia \\
\hline & Congenital syphilis: clinical features and diagnosis \\
\hline Feb-20 & $\begin{array}{l}\text { Epidemiology, clinical presentation, and diagnostic evaluation of } \\
\text { para pneumonic effusion and empyema in adults }\end{array}$ \\
\hline & Achalasia: Pathogenesis, clinical manifestations, and diagnosis \\
\hline & $\begin{array}{l}\text { Definition, classification, etiology, and pathophysiology of shock in } \\
\text { adults }\end{array}$ \\
\hline Dec-19 & Treatment of pheocromocytoma in adults \\
\hline & $\begin{array}{l}\text { Acute complicated urinary tract infection (including pyelonephritis) } \\
\text { in adults }\end{array}$ \\
\hline & Management of locally advanced cervical cancer \\
\hline Oct-19 & Overview of hypertension in adults \\
\hline & $\begin{array}{l}\text { Invasive cervical cancer: Epidemiology, risk factors, clinical } \\
\text { manifestations, and diagnosis }\end{array}$ \\
\hline & Anemia in pregnancy \\
\hline &
\end{tabular}




\section{Access Methods}

Figure 1 shows the number of times the tool was accessed by registered users via the website or the app for each two-month period being reported in the months of October and December 2019, and February, April, June, and August 2020.

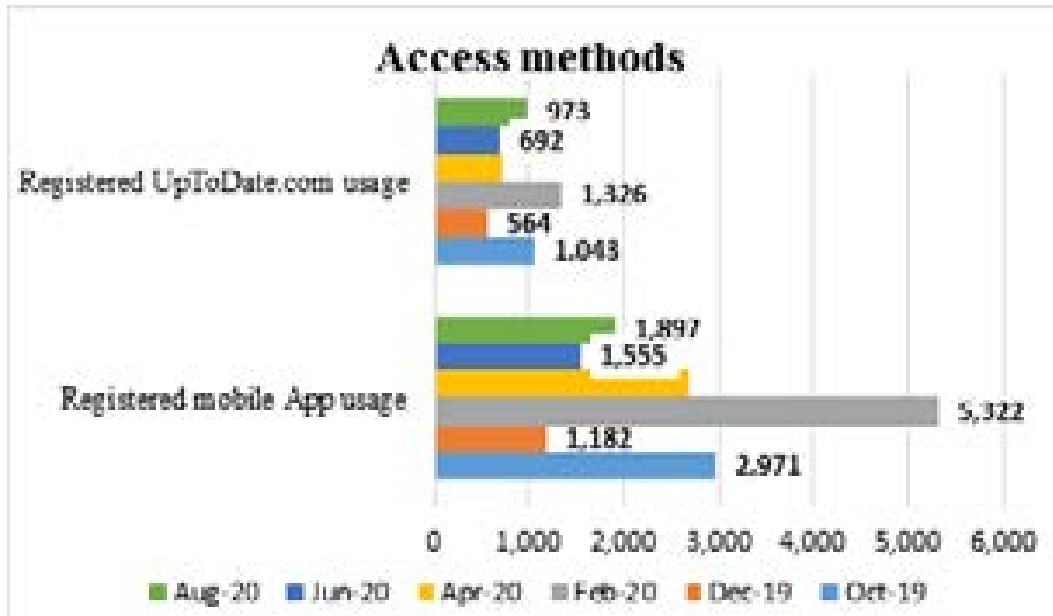

Figure 1: MakCHS: Access methods-Oct and Dec 2019, Feb -Aug 2020

Usage via the appwas consistently about two to three times more common than usage via the website but both forms of usage followed approximately the same trends. The February 2020 report showed the most usage via both the web $(1,326)$ and the app $(5,322)$ while the December 2019 report showed the lowest usage of any two-month period.

\section{User roles}

Table 2 shows the roles of the users who accessed Up-
ToDate by reporting period. People with a range of roles registered for and accessed the tool. There were consistently a higher number of medical students and residents accessing the tool compared to physicians and other user types. February and April reporting periods showed the highest numbers of users accessing the tool across the top three roles, i.e., medical students, residents, and physicians. Physician assistants used the tool much more in the December 2019 reporting period than during any other reporting period.

Table 2: MakCHS: User roles that accessed the topics in Oct 2019, Dec 2019, Feb 2020 and Apr 2020

\begin{tabular}{|l|r|r|r|r|r|r|r|}
\hline & & & & & \multicolumn{2}{|l|}{$\begin{array}{l}\text { June- } \\
\mathbf{2 0}\end{array}$} & $\begin{array}{l}\text { Total } \\
\text { users }\end{array}$ \\
\hline User role & Oct-19 & Dec-19 & Feb-20 & Apr-20 & Ang \\
\hline Medical student & 133 & 64 & 133 & 132 & 48 & 61 & $\mathbf{5 7 1}$ \\
\hline Resident & 46 & 44 & 52 & 52 & 37 & 50 & $\mathbf{2 8 1}$ \\
\hline Physician & 22 & 18 & 27 & 27 & 23 & 27 & $\mathbf{1 2 1}$ \\
\hline Other & 5 & 0 & 1 & 2 & - & 2 & $\mathbf{1 0}$ \\
\hline Medical Librarian & 1 & 1 & 0 & 0 & - & 2 & $\mathbf{2}$ \\
\hline Nurse & 0 & 1 & 1 & 1 & 1 & 1 & $\mathbf{5}$ \\
\hline Pharmacist & 1 & 1 & 2 & 2 & 1 & 1 & $\mathbf{8}$ \\
\hline Physician assistant & 1 & 21 & 1 & 1 & 1 & - & $\mathbf{2 5}$ \\
\hline Nurse practitioner & 0 & 0 & 1 & 1 & 1 & 1 & $\mathbf{4}$ \\
\hline TOTAL & 209 & 150 & 218 & 218 & 112 & 145 & 1,027 \\
\hline
\end{tabular}

Source: Better Evidence

\section{Medical specialties by views}

The topics viewed fall into different specialty areas. The top five topic specialties viewed from October 2019 to August 2020 are shown in Table 3. 
Table 3: MakCHS: Up-To-Date users by specialty during the months of Oct. 2019, Dec. 2019, Feb. 2020, Apr. 2020 and Jun.2020

\begin{tabular}{|c|c|c|c|c|c|c|c|}
\hline $\begin{array}{l}\text { Top Five Topic Specialties } \\
\text { Viewed }\end{array}$ & $\begin{array}{l}\text { Oct- } \\
19\end{array}$ & $\begin{array}{l}\text { Dec- } \\
19\end{array}$ & Feb-20 & $\begin{array}{l}\text { Apr- } \\
20\end{array}$ & Jun20 & Aug-20 & TOTAL \\
\hline Pediatrics & 565 & 171 & 995 & 585 & 352 & 373 & 3,041 \\
\hline $\begin{array}{l}\text { Obstetrics, gynecology \& } \\
\text { women's health }\end{array}$ & 493 & 294 & 762 & 494 & 139 & 399 & 2,581 \\
\hline Infectious diseases & 208 & 153 & 607 & 294 & 135 & 250 & 1,647 \\
\hline Drug information & - & 223 & - & 297 & 172 & 360 & 1,052 \\
\hline $\begin{array}{l}\text { Gastroenterology and } \\
\text { Herpetology }\end{array}$ & - & - & 518 & - & 121 & - & 639 \\
\hline & 1,266 & 841 & 2,882 & 1,670 & 919 & 1,382 & 8,960 \\
\hline
\end{tabular}

Source: Better Evidence

Pediatrics, obstetrics and gynecology \& women's health registered the most topic hits over the course of the year, while gastroenterology and herpetology received a lot of views during two reporting periods.

\section{Medical topics by views}

The top five most viewed medical topics by reporting period are shown in Table 4. The pattern suggests diverse usage. In some reporting periods, there are clear connections between the medical topics viewed, while in other periods there is quite a range of views. In general, the number of views for each specific topic is relatively low compared to the total number of views of all topics.

Table 4: Top Five Medical Topics by views

\begin{tabular}{|l|l|}
\hline & $\begin{array}{l}\text { Overview of the clinical presentation and diagnosis of acute lymphoblastic } \\
\text { leukemia/lymphoma in children (31) } \\
\text { Pelvic organ prolapse in women: Diagnostic evaluation (29) } \\
\text { Pelvic organ prolapse in women: Epidemiology, risk factors, clinical manifestations, } \\
\text { and management (27) } \\
\text { Oct-19 }\end{array}$ \\
\hline Presentation, diagnosis, and staging of Wilms tumor (22) \\
Dec-19 & $\begin{array}{l}\text { Paragangliomas: Epidemiology, clinical presentation, diagnosis, and histology (15) } \\
\text { Spinal anesthesia: Technique (15) }\end{array}$ \\
\hline Intrapartum fetal heart rate assessment (11)
\end{tabular}

Source: Better Evidence 


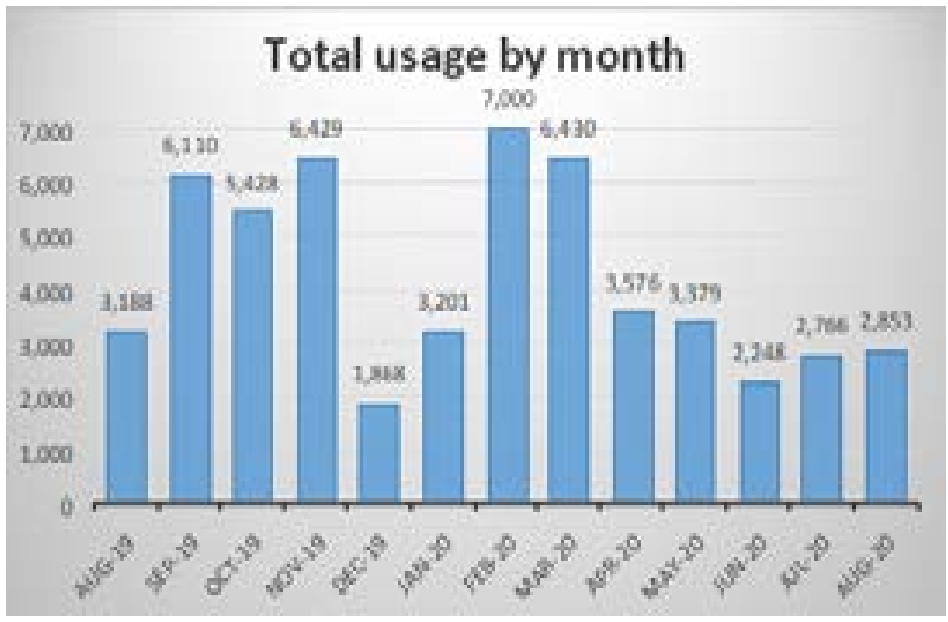

Figure 2: Total usage by month: Aug 2019-Aug 2020

\section{Discussion}

The discussion of findings is presented according to the subheadings discussed in the results section:

Access methods data shows that users primarily accessed UpToDate on mobile devices rather than computers. This suggests that users are able and willing to access the digital tool while on the go and not only in a static location. This is promising given the relevance of the tool for clinical care use at the patient bedside where there are often no computers. This also suggests that people are willing to use their own personal devices for learning and consulting the evidence given the school does not provide mobile devices. Furthermore, hospitals in Uganda, where the students and residents do ward rounds do not necessarily have internet connection, so the fact that use occurred on mobile devices suggests that users may have been able to download the UpToDate content and use the offline feature of the tool. The ability to use the tool offline is likely make the use of UpToDate on mobile devices more appealing and could be an important feature when considering use of digital tools in limited-resource settings.

User role data is promising and it shows those in their training years at the institution used Up-To-Date most. This finding is encouraging as these are critical years for establishing habits for practice for the years ahead. It is important to note however that the denominator for the various groups users groups varies, which may explain why some user groups appear to use the tool less. For example, the college has only three librarians compared to 2,700 students.

The beginning of the semester is characterized by exams and tests, and that is the time when lectures are gaining momentum. Students are therefore usually busy and this could have affected usage. The month of March 2020 was further affected by the lockdown and closure of institutions of higher education in Uganda due to the COVID 19 pandemic. This, coupled with the beginning of semester schedules, could have further affected usage.

Medical specialties and topics by views data shows that pediatrics, obstetrics and gynecology, infectious diseases, drug information, and gastroenterology and herpetology registered many and continuous viewers throughout the period indicated. While the increased views of the infectious diseases specialty during the month of February 2020 could be attributed to the COVID-19 pandemic, the various topic specialties viewed suggest just how one point-of-care tool can be used in a variety of situations that benefit various categories of users. The data also suggest that these few specialties are especially keen on using information that is up to date to facilitate the application of evidence-based medicine in daily clinical practice. The ability to access COVID-19 information without logging in also may have impacted the data on topics and specialties viewed.

Total usage by month data variation can mostly be explained by outside situational factors. For example, MakCHS received access to Up-To-Date in July 2019. Therefore, in August, most potential users were just becoming aware of the availability of the tool, and trainings were just starting. After being introduced to the tool, users started accessing it more. Between July and September, usage increased substantially. Trainings were conducted weekly during this period, suggesting a clear impact between training and usage. Usage then clearly 
decreased during the holiday break but came back up once classes resumed. In March 2020, MakCHS went into lockdown due to the COVID-19 pandemic and as of August 2020, the students were still under lockdown, although remote work had been eased for a few members of staff. The big difference between usage in February and June 2020 was therefore likely a result of the lockdown and closure of the university, due to the COVID-19 pandemic. There were no trainings or awareness campaigns creation during that time, and students were likely to have less internet access while remote as well.

It is probable that, had it not been for the lockdown due to the COVID-19 pandemic at the end of March, usage would have been higher than it was. However, even in the lockdown, the tool is still being accessed and used, though to a smaller extent. This suggests that those who had registered for the tool before the lockdown have continued to use it off campus, likely having formed the habit of use.

\section{Conclusions and recommendations}

Data suggests that though UpToDate is used in a variety of ways by a variety of user types at the university, usage remains relatively low considering the total number of students, faculty, and potential searches. Therefore, there is a need for continued advocacy and capacity building for the various users of the tool and others like librarians, who promote its uptake and usage. As for the librarians, who promote the uptake and usage of the tool, there is need for further capacity building and awareness. The usage trend, however, suggests that, irrespective of what method of access used to access the tool, users had started embracing the tool before the COVID-19 pandemic and closure of institution. Continued use in such difficult times suggests that there is potential for increased and more consistent use in the future.

Increased capacity building and promotion is expected to go a long way in increasing usage of evidence-based digital tools. The Better Evidence for Champions program, launched in August 2020, in which local librarians, faculty members, and ICT professionals are trained to aid in promoting uptake, is one possibility for increasing uptake via trained local advocates. As bi-monthly data collection will continue, further analysis after the launch of the Champions program will be conducted. This project and other similar efforts that aim to promote the use of evidence in clinical care will be critical to improving the quality of care and health outcomes for patients for decades to come.

\section{Limitations of the study}

The demographic characteristics of the respondents were not captured because of the nature of the study. The data was remotely captured based on log ins in the system, and this could not allow for the capture of data, other than the number of $\log$ ins and the topics viewed.

\section{Conflict of interest}

None declared.

\section{References}

1. Shimizu T, Nemoto T, Tokuda Y. Effectiveness of a clinical knowledge support system for reducing diagnostic errors in outpatient care in Japan: a retrospective study. International Journal of Medical Informatics. 2018;109:1-4.

2. Grandview Research. Clinical Decision Support Systems (CDSS) Market Size, Share \& Trends Analysis Report By Product, By Application (Allery Alert, Dosing Support), By Delivery Mode (On-premise, Cloud, Web), By Component, And Segment Forecasts, 2018 2024. 2018 GVR-2-68038-339-3.

3. Association AM. AMA Digital Health Research: Physician's motivation and requirements for adopting digital health-adoption and attitudinal shifts from 2016 to 2019. 2020.

4. UpToDate. UpToDate Homepage [Internet]. UpToDate. 2020 [cited 2020 Sep 3]. Available from: 2020. Available from: http://uptodate.com.

5. Adepoju I-OO, Albersen BJA, De Brouwere V, van Roosmalen J, Zweekhorst M. mHealth for clinical decision-making in sub-Saharan Africa: a scoping review. JMIR mHealth and uHealth. 2017;5(3):e38.

6. Peterson MW, Rowat J, Kreiter C, Mandel J. Medical students' use of information resources: Is the digital age dawning? Academic Medicine. 2004;79(1):89-95.

7. Barteit S, Jahn A, Banda SS, Bärnighausen T, Bowa A, Chileshe $G$, et al. E-learning for medical education in Sub-Saharan Africa and low-resource settings. Journal of Medical Internet Research. 2019;21(1):e12449.

8. Valtis K, Rosenberg JD, Wachter K, Kisenge RM, Chande Mallya R, David WT, et al. Better evidence: prospective cohort study assessing the utility of an evidence-based clinical resource at the University of Rwanda. BMJ Open. 2019;9(8):1-8.

9. Chen C, Buch E, Wassermann T, Frehywot S, Mullan F, Omaswa F, et al. A survey of Sub-Saharan African medical schools. Human Resources for Health. 2012;10(1):4. 10. Ahmadi S-F, Faghankhani M, Javanbakht A, Akbarshahi M, Mirghorbani M, Safarnejad B, et al. A comparison of answer retrieval through four evidence-based 
textbooks (ACP PIER, Essential Evidence Plus, First Consult, and UpToDate): a randomized controlled trial. Medical Teacher. 2011;33(9):724-30.

11. Edson RS, Beckman TJ, West CP, Aronowitz PB, Badgett RG, Feldstein DA, et al. A multi-institutional survey of internal medicine residents' learning habits. Medical Teacher. 2010;32(9):773-5.

12. Rodrigo C, Maduranga S, Withana M, Fernando D,
Rajapakse S. Knowledge seeking behaviours of pre interns and early career doctors in Sri Lanka: A cross sectional study. BMC Research Notes. 2015;8(1):610.

13. University M. Historical background Kampala: Makerere University; 2020. Available from: https://www. mak.ac.ug/about-makerere/historical-background.

14. Sciences MUCoH. College Profile: Makerere University; 2020 [cited 2020]. Available from: http://chs. mak.ac.ug/content/college-profile. 